Substituting this value in (13), we find that the coefficient of the highest power of $x$ in the first member is $-2 \alpha^{3}$. This however cannot vanish on account of the assumption that $\alpha \neq 0$. It follows that there are no functions satisfying condition (9) except when $R=0$. Therefore

No equation of type III, that is of the Riccati type proper, can represent an isothermal system.

The complete answer to the question proposed at the beginning of the paper may now be stated :

If the differential equation of an isothermal system is included in the form $y^{\prime}=P+Q y+R y^{2}$, then the system must belong to one of four species : $1^{\circ}$, a set of parallel straight lines; $2^{\circ}$, a pencil of straight lines; $3^{\circ}$, a system of equilateral hyperbolas with common asymptotes; $4^{\circ}$, a system of logarithmic cosine curves. $y+\log \cos x=$ const., which may also be written $e^{y} \cos x=$ const.

Columbia University, December, 1903.

\title{
ON SOME PROPERTIES OF GROUPS WHOSE ORDERS ARE POWERS OF A PRIME.
}

BY DR. W. B. FITE.

(Read before the American Mathematical Society, December 28, 1903.)

In the Transactions, volume 3 (1902), page 334, the writer has shown that if a metabelian group of order $p^{m}$, where $p$ is a prime, contains an abelian subgroup of order $p^{m-a}$, the $p^{a}$ power of every operator is invariant. In the same article, page 349, it was shown that a metabelian group of odd order cannot be a group of cogredient isomorphisms if it has a set of generators such that the order of any one of them is not a divisor of the least common multiple of the orders of all the others. This latter was generalized somewhat in the BULLETIN, $2 \mathrm{~d}$ series, volume 9 (1902), page 140. It is the purpose of the present article to carry this generalization somewhat further and to show that the first theorem is a special form of a theorem applicable to all groups whose orders are powers of a prime.

Let $G$ be a group of order $p^{m}$ and class $k$ that contains an abelian subgroup $G_{1}$ of order $p^{m-1}$. If $A$ is any operator of $G_{1}$ and $B$ any operator of $G$ not contained in $G_{1}$, we have 
where

$$
B^{-1} A B=A t_{1}
$$

$B^{-p} A B^{p}=A t_{1}^{p} t_{2}^{\frac{p(p-1)}{2}} \cdots t_{i}^{\frac{p(p-1) \ldots(p-i+1)}{i !}} \cdots t_{p}=A$,

$$
B^{-1} t_{i} B=t_{i} t_{i+1} \quad(i=1,2, \ldots, k-1),
$$

since $B^{p}$ is contained in $G_{1}$. Also

$$
B^{-p} t_{i} B^{p}=t_{i} t_{i+1}^{\frac{p(p-1)}{2}} \cdots t_{i+j}^{\frac{p(p-1) \ldots(p-j)}{(j+1) !}} \cdots t_{i+p}=t_{i} .
$$

The exponents of the successive commutators are the binomial coefficients, and $t_{j}=1$ when $j>k-1$. By giving to $i$ in the equation above the successive values from $k-2$ to $k-p$ inclusive, we see that

$$
t_{j}^{p}=1(j>k-p) .
$$

And in general $t_{j}^{p s}=1$ if $j>k-1-s(p-1)$. Hence $t_{1}^{p r}=1$, if $1>k-1-r(p-1)$ or

$$
r(p-1) \geqq k-1 \text {. }
$$

Therefore $A^{p r}$ is invariant in $G$, and the $p^{r}$ power of every operator is invariant.

Conversely, if $s(p-1)<k-1$, the $\mathrm{p}^{8}$ power of every operator is not invariant. For otherwise

if

$$
t_{i}^{p-j}=1 \text {, }
$$

$$
j(p-1)<i \quad(j=0,1,2, \ldots, s) .
$$

From this it follows that $t_{i}=1$, if $s(p-1)<i$. Hence $t_{k-1}=1$, and $t_{k-2}$ is invariant in $G$. That is, the $(k-2)$ nd adjoined group is abelian, and $G$ is of class $l$, where $l<k$. The results thus obtained can be formulated into the

TheOREM : If a group $G$ of order $p^{m}$ ( $p$ a prime) and class $k$ contains an abelian subgroup of order $p^{m-1}$, the $p^{r}$ power of every operator is invariant if $r(p-1) \geqq k-1$. Conversely, if $G$ contains an abelian subgroup of order $p^{m-1}$ and if the $p^{r}$ power of every operator is invariant, $G$ is of class $k$, where $k-1 \leqq r(p-1)$.

We concern ourselves now with the case in which the abelian subgroup is of order $p^{m-a}$. Let this subgroup be denoted by $G_{1}$ and let $G_{2}$ be a subgroup of $G$ of order $p^{m-a+1}$ that contains 
$G_{1}$, and $G_{3}$ a subgroup of order $p^{m-a+2}$ that contains $G_{2}$. If $B$ is an operator of $G_{3}$ that is not in $G_{2}, B^{p^{r+1}}$ is invariant in $G_{3}$. If $A$ is any operator of $G_{2}$ that is not in $G_{1}, A^{p}$ is invariant in $G_{2}$. Now

where

$$
\begin{aligned}
B^{-1} A^{p} B & =A^{p} t_{1}, \\
B^{-p} A^{p} B^{p} & =A^{p} t_{1}^{p} \cdots t_{i}^{\frac{p(p-1) \ldots(p-i+1)}{i !}} \cdots t_{p}=A^{p},
\end{aligned}
$$

$$
B^{-1} t_{i} B=t_{i} t_{i+1} \quad(i=1,2, \ldots, k-1) .
$$

The commutators $t_{i}$ are invariant in $G_{2}$ since $A^{p}$ is.

As before, it follows that $t_{1}^{p^{r}}=1$, where $r(p-1) \geqq k-1$. Hence

$$
B^{-1} A^{p^{r+1}} B=A^{p^{r+1}} t_{1}^{p^{r}}=A^{p^{r+1}},
$$

and $A^{p^{r+1}}$ is invariant in $G_{3}$. If $C$ is any operator of $G_{1}$, then $C^{p^{r}}$ is invariant in $G_{2}$, and hence $C^{p^{2 r}}$ is invariant in $G_{3}$.

We have therefore shown that the $p^{2 r}$ power of every operator of $G_{3}$ is invariant. Now by induction and a repetition of the argument just used we reach the

THEOREM : If a group $G$ of order $p^{m}$ and class $k$ contains an abelian subgroup of order $p^{m-a}$, the $p^{\text {ra }}$ power of every operator is invariant, where $r(p-1) \geqq k-1$.

Returning now to the case in which the abelian subgroup is of order $p^{m-1}$, we see that if $G_{1}$ is of type $\left(m_{1}, m_{2}, \ldots, m_{n}\right)$, where $l$ of the quantities $m_{i}$ are equal to 1 , then $k \leqq l+2$ or $>p$. For the commutators of $G$ are all contained in $G_{1}$ and if $k \leqq p$ they are all of order $p$. Furthermore, the $p$ th power of every operator is invariant. Hence the order of the commutator subgroup of $G^{\prime}$, the group of cogredient isomorphisms of $G$, is less than, or equal to, $p^{l}$, and $G^{\prime}$ is of class $k^{\prime}$, where $k^{\prime} \leqq l+1 . * \quad$ Therefore $k \leqq l+2$. In particular, if $l=0$, either $G$ is metabelian, or $k>p$.

Suppose now that $G$ is of order $p^{m}$ and class $k$ and that $B^{\prime}$ is the operator of $G^{\prime}$ that corresponds to $B$ of $G . \dagger$ If $B^{\prime}$ is of order $p^{\beta}$ and $A$ is any operator of $G$, we have, if the commutator subgroup of $G$ is abelian,

* Transactions, vol. 3 (1902), p. 350.

† Loc. cit., p. 332 . 


$$
\begin{aligned}
& B^{-p^{\beta}} A B^{p^{\beta}} \\
& =A t_{1}^{p^{\beta}} \cdots t_{j}^{p^{\beta(}\left(p^{\beta}-1\right) \ldots\left(p^{\beta}-j+1\right) / j !} \cdots t_{k-1}^{p^{\beta}\left(p^{\beta}-1\right) \ldots\left(p^{\beta}-k+2\right) /(k-1) !} \\
& =A \text {, } \\
& B^{-p^{\beta}} t_{i} B^{p^{\beta}}=t_{i} t_{i+1}^{p^{\beta}} \cdots t_{k-1}^{p^{\beta}\left(p^{\beta}-1\right) \cdots\left(p^{\beta}-k+i+2\right) /(k-1) !}=t_{i}, \\
& (i=1,2, \ldots, k-2) \text {. }
\end{aligned}
$$

Since $t_{i}^{\beta{ }^{\beta+j}}=1$, when $(j+1)(p-1) \geqq k-i$, it follows that $t_{1}^{\beta+r}=1$ when $(r+1)(p-1) \geqq k-1$.

In a similar way it can be seen that

$$
t_{1}^{\alpha+r}=1,
$$

where $p^{\alpha}$ is the order of $A^{\prime}$. Hence $t_{i}^{p+r}=1$, since

$$
B^{-1} t_{i-1}^{p^{\alpha+r}} B=t_{i}^{p_{1}^{\alpha+r}} t_{i}^{p^{\alpha+r}}=t_{i-1}^{p^{\alpha+r}} \quad(i=\dot{2}, 3, \ldots, k-1),
$$

when

$$
t_{i-1}^{p^{a+r}}=1
$$

But we have just seen that

$$
t_{1}^{p+r}=1 .
$$

Suppose now that $k-s$ is the greatest multiple of $p-1$ less than $k-1$. Then since $G^{(s-1)}$, the $(s-1)$ th adjoined group of $G$, is of class $k-s+1$ and $A_{(s-1)}^{p^{a}}$ is commutative with every operator of $G^{(s-1)}$, we have $t_{1}^{p_{(s-1)}^{a+r}}=1$, where

$$
\left(r^{\prime}+1\right)(p-1)=k-s .
$$

Obviously $r^{\prime}<r$. Now

$$
B^{-1}{ }^{(s-2)} t_{1}^{(s-2} B^{(s-2)}=t_{1}^{(s-2)} t_{2}^{(s-2)} .
$$

But since $t_{1}^{p+s-1)}=1$, we have $t_{2}^{p+r-2)}=1$.

By continuing this reasoning we get $t_{s}^{p^{\alpha+r^{\prime}}}=1$. Hence $t_{i}^{p a+r^{\prime}}=1$, when $i>s$. More generally, we have in $G^{\{s+l(p-1)-1\}}$ the invariant operator

$$
A^{p}{ }_{\{s+l(p-1)-1\}}^{\alpha} .
$$


Hence

$$
t_{1}^{\left.p a+r_{1}+l(p-1)-1\right\}}=1
$$

where $r_{1}=r^{\prime}-l$. Then $t_{i}^{p^{\alpha+\tau_{1}}}=1$, when $i=s+l(p-1)$.

This is equivalent to the condition

$$
\left(r_{1}+1\right)(p-1) \geqq k-i .
$$

We conclude from this that

$$
B^{-p_{\alpha+r}} A B^{p^{\alpha+r}}=A \text {. }
$$

Therefore if $A^{\prime}$ is of order $p^{a}$, the $p^{\alpha+r}$ power of every operator of $G$ is commutative with $A$, when

$$
(r+1)(p-1) \geqq k-1 .
$$

From this result follows the

THeORem : If a group of order $p^{m}$ and class $k$ has an abelian commutator subgroup, its group of cogredient isomorphisms cannot have a set of generators such that the order of any one of them is greater than $p^{r}$ times the order of every one of the others, $r$ being defined by the relation $(r+1)(p-1) \geqq k-1$.

We add a formula for the $n$th power of the product of any two operators of a group of the 3 d class. Let $A$ and $B$ be these operators. Then if

$$
B^{-1} A B=A t_{1}, \quad B^{-1} t_{1} B=t_{1} t_{2}, \quad A^{-1} t_{1} A=t_{1} h,
$$

we have

$$
(B A)^{n}=B^{n} A^{n} t_{1}^{\frac{n(n-1)}{2 !}} t_{2}^{\frac{n(n-1)(n-2)}{3 !}} h^{\frac{n(n-1)(2 n-1)}{3 !}} .
$$

Cornell University,

December, 1903. 